\title{
CLIMABR Parte I: Modelo para a geração de séries sintéticas de precipitação ${ }^{1}$
}

\author{
Vicente de P. S. de Oliveira ${ }^{2}$, Sidney S. Zanetti ${ }^{3}$ \& Fernando F. Pruski ${ }^{4}$
}

\begin{abstract}
${ }^{1}$ Parte da Tese do primeiro autor, apresentada no Programa de Pós-graduação em Engenharia Agrícola na UFV. Viçosa, MG ${ }^{2}$ NPGA/CEFET CAMPOS. Rua Dr. Siqueira 273, Parque Dom Bosco, CEP 28030-130, Campos dos Goytacazes, RJ. Fone: (22) 2733-3255, R 4240. E-mail: vsantos@cefetcampos.br

${ }^{3}$ UENF/CCTA/LEAG. Av. Alberto Lamego 2000, Parque Califórnia, CEP 28013-602, Campos dos Goytacazes, RJ. Fone: (22) 2726-1543. E-mail: sidney@uenf.br

${ }^{4}$ UFV/DEA. Campus Universitário, CEP 36571-000, Viçosa, MG. Fone: (31) 3899-1912. E-mail: ffpruski@ufv.br
\end{abstract}

Protocolo $163-16 / 2 / 2004$ - Aprovado em 5/4/2005

\begin{abstract}
Resumo: Na área agrícola tem-se muitos dos estudos relativos à precipitação buscando avaliar os efeitos advindos da sua ocorrência no escoamento superficial; neste caso, é imprescindível o conhecimento não apenas das informações relativas à lâmina precipitada, à duração e intensidade das chuvas mas, também, ao perfil da precipitação. Objetivou-se, através do presente trabalho, mostrar o desenvolvimento de um modelo denominado CLIMABR, para a geração de séries sintéticas de precipitação, para as condições climáticas encontradas no Estado do Rio de Janeiro; para isto, utilizaram-se dados pluviométricos e pluviográficos de 11 estações da Fundação Superintendência Estadual de Rios e Lagoas - SERLA, e da Companhia LIGHT - Serviços de Eletricidade S.A. Na série sintética gerada obtiveram-se os valores mensais da probabilidade de um dia ser chuvoso tendo sido o anterior também chuvoso $\mathrm{P}(\mathrm{W} / \mathrm{W})$ e da probabilidade de um dia ser chuvoso tendo sido o anterior seco $\mathrm{P}(\mathrm{W} / \mathrm{D})$; o número de dias chuvosos (NW) e a precipitação total diária (P) ocorrida nesses dias, além de outras informações relativas ao perfil de precipitação. A comparação dos dados gerados pelo CLIMABR com os dados observados mostrou um bom desempenho do modelo para a estimativa destas variáveis.
\end{abstract}

Palavras-chave: Chuvas intensas, modelo hidrológico, gerador climático

\section{CLIMABR Part I: Model for generation of synthetic series of precipitation}

\begin{abstract}
In the agricultural area, many studies related to precipitation have tried to evaluate the effects resulting from its occurrence in surface runoff. In this case the knowledge of not only the information relative to precipitation, its duration and intensity of the rains, but, also the profile of the precipitation is of great importance. With this objective, the present work was carried out to develop a model called CLIMABR, for the generation of synthetic precipitation series and of the instantaneous profile associated to these for the climatic conditions of the State of Rio de Janeiro. Pluviometric and pluviographic data from eleven stations of the Fundação Superintendência Estadual de Rios e Lagoas - SERLA and of Companhia LIGHT - Serviços de Eletricidade S.A. were utilized. In the generated synthetic series, the number of rainy days were obtained, according to the probabilities associated to the Markov chain and the total daily precipitation. The comparison of the data generated by CLIMABR with the observed data showed a good performance of the model for the estimation of these variables.
\end{abstract}

Key words: intensive rainfall, hydrologic model, climate generator

\section{INTRODUÇÃO}

O estudo do clima vem recebendo atenção especial do homem, ao longo do tempo, não só por influenciar diretamente no seu cotidiano mas, sobretudo, por ser um dos principais fatores que afeta a produção de alimentos. Nos últimos anos, a humanidade despertou para as ameaças que rodeiam, em decorrência do uso indiscriminado dos recursos naturais, o que tem provocado a degradação do ar, da água e do solo, causando profundas transformações ambientais, em particular no clima do planeta.

A contínua e acelerada degradação ambiental praticada pelo homem é provocada por vários fatores, dentre os quais se 
destacam: os desmatamentos, que buscam a ampliação das fronteiras agrícolas, provocando a destruição das nascentes e o assoreamento dos cursos d'água; a ocupação desordenada das margens dos cursos d'água; uso inadequado do solo na área agrícola, que tem proporcionado um aumento das vazões máximas, causando enchentes cada vez mais freqüentes, e a redução das vazões mínimas, causando grandes problemas de abastecimento e de geração de energia além da utilização irracional da água pelos diversos usuários, gerando os conflitos devido, ao uso exagerado. A interação destes fatores, associada à necessidade de estimativas mais adequadas das precipitações, a fim de reduzir os prejuízos causados pelos eventos extremos, tem provocado um crescente interesse no estudo deste elemento climático.

Dos fatores citados, muitos estão relacionados com a erosão hídrica, processo decorrente do desprendimento, transporte e deposição das partículas do solo causados pelo impacto das gotas da chuva e pelo escoamento superficial. O conhecimento das características das chuvas, principalmente dos totais precipitados e da variação da sua intensidade ao longo dos eventos é fundamental para uma adequada representação destes processos. As pesquisas nesta área têm evoluído bastante, sobretudo em função do uso de técnicas de simulação. Esta ferramenta, utilizada amplamente no meio científico, tem auxiliado pesquisadores e profissionais na avaliação dos impactos ambientais advindos do uso de sistemas de preparo e manejo do solo, bem como no dimensionamento de obras hidráulicas e no gerenciamento de recursos hídricos.

O emprego de dados obtidos a partir de geradores de precipitação em modelos de simulação hidrológica, principalmente naqueles utilizados na predição das taxas de escoamento superficial e de perdas de solo, tem-se intensificado nos últimos anos. Esses geradores, normalmente desenvolvidos a partir de séries históricas disponíveis, são avaliados em função da capacidade que apresentam para gerar dados com estatísticas similares às observadas nos dados históricos. O modelo CLIGEN - CLImate GENeration, desenvolvido por Nicks et al. (1995), usa estatísticas de dados climáticos históricos, observados em estações meteorológicas, com o propósito de gerar séries temporais de dados. O modelo foi desenvolvido a partir de um conjunto de 1078 arquivos de parâmetros de várias estações, para 50 estados e territórios dos Estados Unidos (Flanagan et al., 2001).

No Brasil, poucos trabalhos de pesquisa têm sido direcionados para a obtenção de modelos que possibilitem a geração de dados climáticos. Normalmente as pesquisas se limitam ao estudo de freqüência das séries históricas disponíveis ou a ajustes de funções de densidade de probabilidade a esses dados. Entendendo a necessidade e a importância da disponibilização de modelos que permitam a geração de dados de precipitação para serem utilizados em diversas áreas, em particular em aplicações ligadas à agricultura e no dimensionamento de obras hidráulicas, realizou-se este trabalho, com o objetivo de desenvolver um modelo para geração de séries sintéticas de precipitação com o qual é possível obter, para cada dia chuvoso, as seguintes informações: os valores mensais da probabilidade de um dia ser chuvoso, tendo sido o anterior também chuvoso $\mathrm{P}(\mathrm{W} / \mathrm{W})$ e da probabilidade de um dia ser chuvoso tendo sido o anterior seco P(W/D); enfim, o número de dias chuvosos NW e a precipitação total diária (P) ocorrida nestes dias, além de outras informações relativas ao perfil de precipitação.

\section{MATERIAL E MÉTODOS}

O CLIMABR foi desenvolvido com base na premissa de que, para cada dia chuvoso, ocorreu um único evento, o qual é estatisticamente equivalente ao efeito combinado das diversas precipitações que, eventualmente, tenham ocorrido neste dia. Para a utilização do modelo CLIMABR são necessários, efetivamente, somente dados pluviométricos.

O desenvolvimento do modelo foi possível a partir de informações de 11 estações meteorológicas situadas no Estado do Rio de Janeiro e operadas pela Fundação Superintendência Estadual de Rios e Lagoas - SERLA, e pela Companhia LIGHT - Serviços de Eletricidade S.A. Utilizaram-se dados originários de pluviogramas e de pluviômetros. As informações sobre as estações estudadas são apresentadas no Tabela 1, e a localização das estações consta na Figura 1.

Os dados pluviométricos já se encontravam consistidos e organizados em planilhas e em arquivos texto, enquanto que os dados pluviográficos foram digitalizados diretamente a partir dos pluviogramas.

A média, o desvio padrão e o coeficiente de assimetria da precipitação total diária correspondente aos dias chuvosos, além da probabilidade de um dia ser chuvoso tendo sido o anterior também chuvoso e da probabilidade de um dia ser chuvoso tendo sido o anterior seco, foram obtidos a partir dos dados pluviométricos.

Para a definição da ocorrência ou não de precipitação num dado dia foi gerado, inicial e internamente, no modelo CLIMABR, um conjunto de números aleatórios, que variou de 0 a 1 , sendo a sua extensão igual ao número de dias da série sintética a ser gerada. Utilizou-se o procedimento de obter sempre o mesmo conjunto de números aleatórios, a fim de que se obtivessem resultados iguais quando da realização das simulações em uma mesma estação favorecendo, desta forma, o uso do modelo em estudos científicos, uma vez que, passa a permitir a obtenção de reprodutibilidade dos resultados, sob condições idênticas de simulação.

As probabilidades condicionais da cadeia de Markov de $1^{\text {a }}$ ordem, empregadas neste estudo foram descritas por Nicks et al. (1995) conforme as equações:

$$
\begin{gathered}
\mathrm{P}(\mathrm{W} / \mathrm{D})=\frac{N W D}{N D} \\
\mathrm{P}(\mathrm{W} / \mathrm{W})=\frac{N W W}{N W}
\end{gathered}
$$

em que:

$\mathrm{P}(\mathrm{W} / \mathrm{D})$ - probabilidade, obtida para cada mês, de um dia ser chuvoso, tendo sido o anterior seco, decimal 


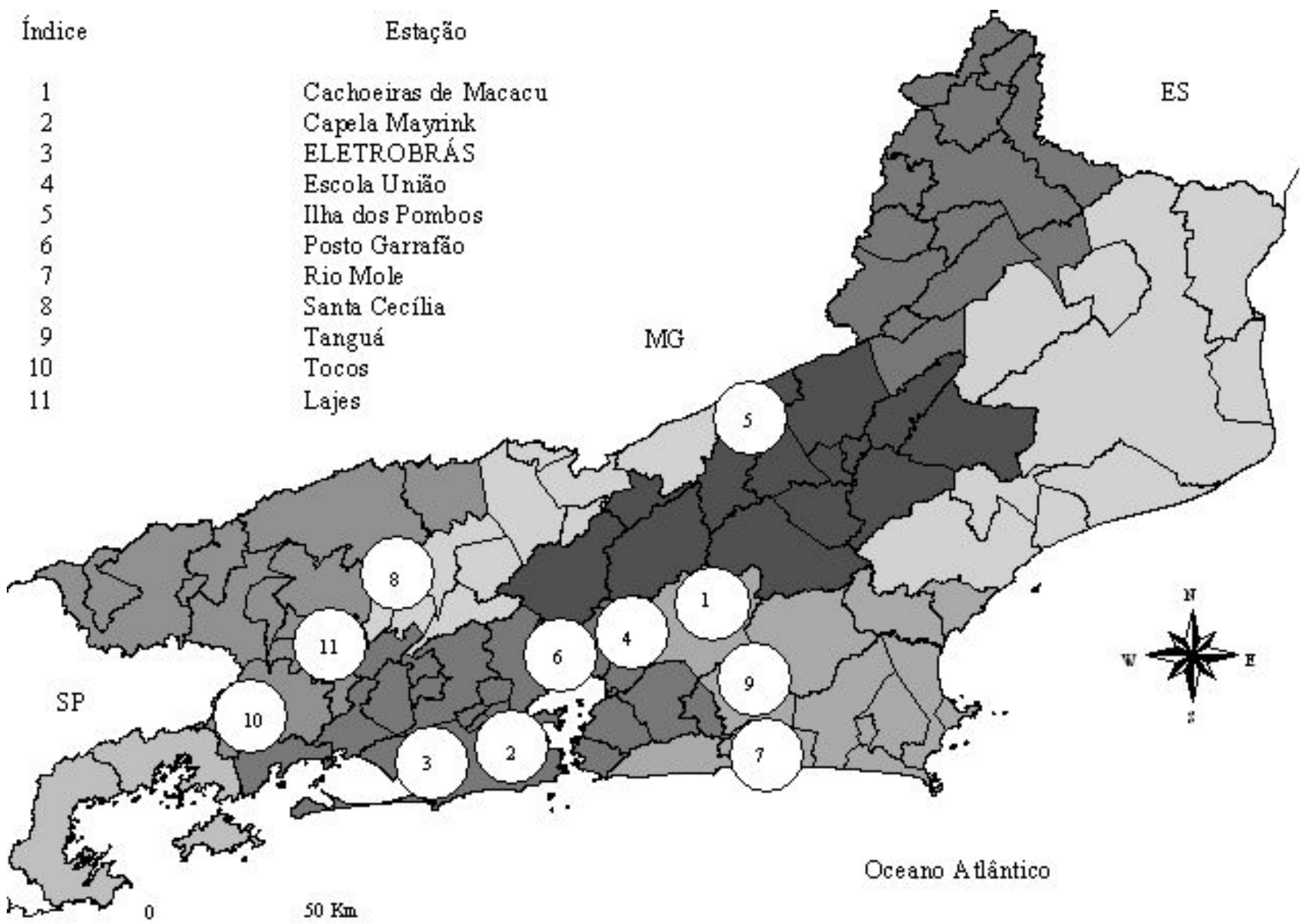

Figura 1. Localização das estações meteorológicas do Estado do Rio de Janeiro utilizadas no desenvolvimento do modelo CLIMABR

Tabela 1. Informações relativas às estações meteorológicas, situadas no Estado do Rio de Janeiro, utilizadas como base de dados para o desenvolvimento do modelo CLIMABR

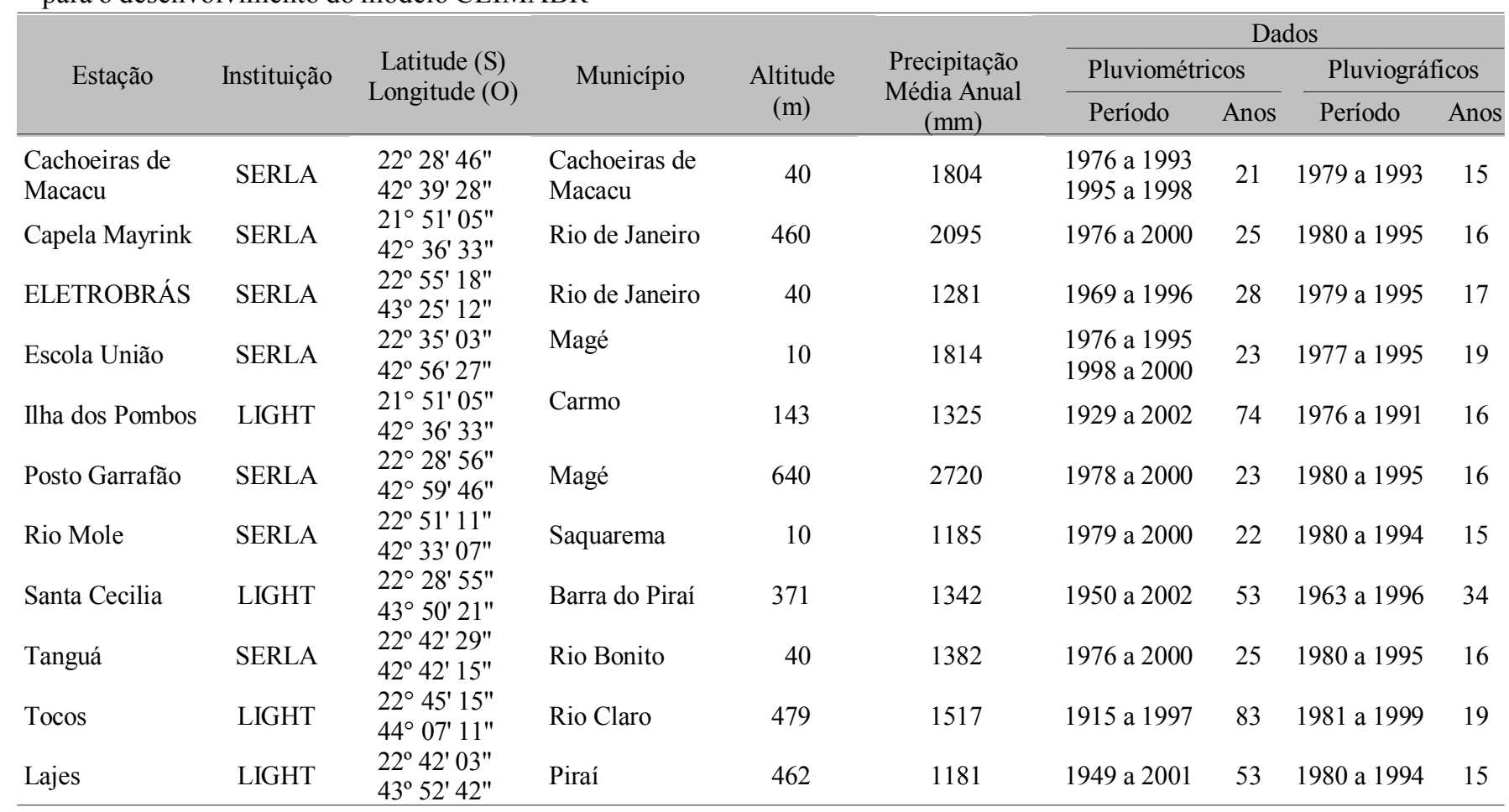


NWD - número de dias chuvosos do mês tendo sido o anterior seco, dias

ND - número de dias secos no mês, dias

$\mathrm{P}(\mathrm{W} / \mathrm{W})$ - probabilidade, obtida para cada mês, de um dia ser chuvoso tendo sido o anterior também chuvoso, decimal

NWW - número de dias chuvosos do mês, tendo sido o anterior chuvoso, dias

NW - número de dias chuvosos no mês, dias

A probabilidade de ocorrência de um dia chuvoso foi calculada, para cada mês, pela equação:

$$
\mathrm{P}(\mathrm{W})=\frac{\mathrm{NW}}{\mathrm{NDM}}
$$

em que NDM é o número de dias do mês, dias.

Optou-se, neste estudo, pela realização dos cálculos e análise dos resultados considerando os valores médios mensais das variáveis estudadas, conforme metodologia adotada por diversos autores (Flanagan et al., 2001; Elliot \& Arnold, 2001).

Para os dias chuvosos, a precipitação total diária foi estimada a partir de uma equação originada da distribuição Pearson tipo III, cujos parâmetros utilizados (média, desvio padrão e coeficiente de assimetria) foram gerados dos valores médios mensais da série histórica. A precipitação total diária foi calculada por:

$$
P^{\prime}=\mu+\frac{2 s}{g}\left(\left(\frac{g}{6}\left(x^{\prime}-\frac{g}{6}\right)+1\right)^{3}-1\right)
$$

em que:

P' - precipitação total diária, $\mathrm{mm}$

$\mu$ - média mensal da precipitação total diária, mm

s - desvio padrão da precipitação total diária, $\mathrm{mm}$

g - coeficiente de assimetria da precipitação total diária, adimensional

x’ - variável normal padrão, adimensional

Os valores diários da variável normal padrão utilizados na Eq. 4, foram obtidos a partir da padronização de um novo conjunto de números aleatórios, gerados no modelo.

Após a geração da precipitação total diária para a série, o CLIMABR realiza uma correção nas médias mensais e nos desvios padrão, de forma a reduzir as diferenças entre os valores gerados e os observados. A correção da média é efetuada por:

$$
\mathrm{Pc}=\frac{\mu}{\mu_{\mathrm{c}}} \mathrm{P}^{\prime}
$$

em que:

Pc - precipitação total diária com média corrigida, $\mathrm{mm}$

$\mu_{\mathrm{c}}$ - média mensal da precipitação total diária calculada, $\mathrm{mm}$
A correção do desvio padrão é obtida por:

$$
\mathrm{P}=(\mathrm{Pc}-\mu) \frac{\mathrm{S}}{\mathrm{S}_{\mathrm{c}}}+\mu
$$

em que $S_{c}$ é o desvio padrão da precipitação total diária calculada a partir da série sintética.

Geraram-se séries sintéticas para 100 anos tanto a partir do CLIGEN como pelo CLIMABR neste trabalho. O CLIGEN requer, como dados de entrada, além das informações gerais, as seguintes informações sobre a precipitação: a média dos valores diários máximos da intensidade da precipitação em 30 min (por mês); a média, o desvio padrão e o coeficiente de assimetria da precipitação total diária (por mês); as probabilidades de um dia ser chuvoso, tendo sido o anterior seco ou chuvoso (por mês); precipitação máxima em $30 \mathrm{~min}$ (por mês); e os valores da curva de distribuição acumulada do tempo de ocorrência da intensidade máxima instantânea de precipitação.

Os resultados foram apresentados e discutidos em termos de médias mensais, correspondentes ao número de dias chuvosos, à probabilidade de um dia ser chuvoso tendo sido o anterior também chuvoso, à probabilidade de um dia ser chuvoso e o anterior seco e à precipitação total diária.

A partir dos dois modelos, os resultados encontrados foram também apresentados na forma de gráficos e tabelas, a fim de permitir uma comparação visual entre os resultados apresentados pelo CLIMABR e no CLIGEN, e os dados observados. Objetivando-se avaliar a tendência das 12 médias mensais simuladas ao longo do ano, ajustaram-se também equações de regressão linear simples para verificação da dispersão das médias mensais simuladas em relação às médias mensais observadas. A verificação dos resultados obtidos foi realizada com base na análise dos gráficos de dispersão, por meio do coeficiente de determinação $\left(\mathrm{r}^{2}\right)$ das regressões, permitindo, assim, uma avaliação da correlação entre os resultados obtidos pelo modelo e os dados observados.

Foram também, calculadas, as variações percentuais $(\delta)$ entre as médias mensais geradas pelos modelos e os valores observados nas estações. A partir do módulo dos valores das variações percentuais $(\delta)$ calculou-se, para cada estação, o valor médio das variações percentuais (VMVP), o que permitiu a comparação entre os valores gerados pelos modelos CLIMABR e CLIGEN e entre as estações estudadas.

Utilizou-se, também, o cálculo do intervalo de confiança, em um nível de 95\% de significância, para as médias mensais, com o objetivo de se avaliar, estatisticamente, os valores gerados, através do uso do gráfico de barras.

\section{RESULTADOS E DISCUSSÃO}

\section{Número de dias chuvosos - NW}

Constatou-se que, de maneira geral, o CLIMABR e o CLIGEN apresentaram maiores variações percentuais em relação aos valores constatados nos desvios padrão do que nas médias mensais, para esta variável. Outro comportamento 
evidenciado foi a tendência de obtenção de valores, seja pelo CLIMABR ou pelo CLIGEN, inferiores aos observados. Esta tendência foi notada também para a média do número médio de dias chuvosos, como em relação ao desvio padrão.

Os resultados relativos aos valores médios mensais do número de dias chuvosos e dos respectivos desvios padrão dos dados gerados pelo CLIMABR e pelo CLIGEN, as variações percentuais em relação aos dados observados e o valor médio dessas variações, além dos próprios dados observados na estação Cachoeiras de Macacu, encontra-se na Tabela 2.

Com relação aos resultados gerados pelo modelo CLIMABR na estação Cachoeiras de Macacu, as médias mensais apresentaram variações de $-1,7 \%$ (setembro) a $8,5 \%$ (maio). Os desvios padrão apresentaram variações percentuais que oscilaram entre uma superestimativa de $22,2 \%$ (abril) a uma subestimativa de $-45,3 \%$ (março).

As variações percentuais entre os resultados obtidos utilizando-se o CLIGEN e os valores observados, apresentaram amplitude semelhante, variando de $-2,8 \%$ (julho) a $4,2 \%$ (abril). Os desvios padrão obtidos pelo CLIGEN apresentaram variações percentuais em relação aos dados observados que oscilaram de $-45,3 \%$ (março), até 33,3\%, o que ocorreu no mês de abril.

Dos valores médios das variações percentuais (VMVP) obtidos para o CLIMABR e para o CLIGEN, os resultados obtidos nesta estação foram próximos, observando-se maior variação em termos de desvio-padrão para o CLIGEN.

Outros trabalhos consultados também apresentam variações de magnitude similar ou superior às variações evidenciadas neste trabalho, para o número de dias chuvosos. Virgens Filho (1997) desenvolveu um modelo, chamado GEPAC, em que o número de dias chuvosos foi superestimado para quatro localidades do Estado de São Paulo. As variações percentuais observadas alcançaram valores de $12,0 \%$ no mês de agosto para Botucatu, 17,0\% em novembro para Campos do Jordão, 23,0\% em agosto para Pontal e 24,0\% em setembro para Votuporanga. Virgens Filho (2001) ainda determinou o numero de dias chuvosos utilizando este mesmo modelo, sendo que os resultados obtidos, para 30 anos de simulação variaram em até $43,0 \%$, em relação aos dados observados.

$\mathrm{Na}$ comparação dos resultados obtidos para as estações localizadas em regiões próximas ao litoral (Cachoeiras de Macacu, ELETROBRÁS, Escola União, Rio Mole e Tanguá) com os gerados nas estações localizadas em maiores altitudes (Capela Mayrink, Ilha dos Pombos, Posto Garrafão, Santa Cecília, Tocos e Lajes) foram constatados comportamentos diferenciados nos resultados, principalmente em relação às médias geradas pelos modelos. Os maiores valores de VMVP para as médias mensais geradas pelo CLIMABR ocorreram nas estações localizadas em maiores altitudes, tendência também que se repetiu nos VMVP's das médias mensais e desvios padrão geradas pelo CLIGEN. Com relação aos desvios padrão gerados pelo CLIMABR, os maiores valores obtidos também ocorreram para estações localizadas em maiores altitudes (Lajes e Santa Cecília), entretanto, para as demais estações houve uma alternância na ordem desses valores. Nitidamente, observou-se que as estações Santa Cecília e Lajes foram as que apresentaram os maiores valores de VMVP para as médias e desvios padrão, tanto no CLIMABR quanto no CLIGEN.

O desempenho diferenciado na geração de dados de precipitação em regiões com maiores altitudes já foi constatado por outros autores (Wilks, 1999; Yu, 2001). Este comportamento pode ser explicado pela predominância de chuvas de formação orográfica nestas regiões, proporcionando uma grande variabilidade com relação à ocorrência de eventos de precipitação.

\section{Probabilidades condicionais da cadeia de Markov}

Probabilidade de um dia ser chuvoso tendo sido o anterior também chuvoso - $\mathbf{P}(\mathbf{W} / \mathbf{W})$ : Evidenciou-se, mais uma vez, de uma maneira geral, que tanto o CLIMABR e o CLIGEN, apresentaram maiores variações percentuais em relação aos valores dos desvios padrão do que nas médias mensais; outro comportamento constatado foi a tendência de obtenção de valores, seja pelo CLIMABR ou pelo CLIGEN, inferiores aos observados. Esta tendência também foi verificada tanto para a

Tabela 2. Valores médios mensais do número de dias chuvosos ( $\overline{\mathrm{NW}}$ ) e os respectivos desvios padrão (s) dos dados observados e gerados pelo CLIMABR e pelo CLIGEN, variações percentuais $(\delta)$ em relação aos dados observados e o valor médio das variações percentuais (VMVP) para a estação Cachoeiras de Macacu

\begin{tabular}{|c|c|c|c|c|c|c|c|c|c|c|}
\hline \multirow[b]{2}{*}{ Meses } & \multicolumn{2}{|c|}{ Observado } & \multicolumn{4}{|c|}{ CLIMABR } & \multicolumn{4}{|c|}{ CLIGEN } \\
\hline & $\begin{array}{l}\overline{\mathrm{NW}} \\
\text { (dias) }\end{array}$ & $\begin{array}{c}\mathrm{s} \\
\text { (dias) }\end{array}$ & $\begin{array}{c}\overline{\mathrm{NW}} \\
\text { (dias) }\end{array}$ & $\begin{array}{c}\delta \\
\%\end{array}$ & $\begin{array}{c}\mathrm{s} \\
\text { (dias) }\end{array}$ & $\begin{array}{c}\delta \\
\%\end{array}$ & $\begin{array}{l}\overline{\mathrm{NW}} \\
\text { (dias) }\end{array}$ & $\begin{array}{l}\delta \\
\%\end{array}$ & $\begin{array}{c}\mathrm{s} \\
\text { (dias) }\end{array}$ & $\begin{array}{c}\delta \\
\%\end{array}$ \\
\hline Jan & 16,9 & 5,2 & 16,9 & 0,0 & 4,1 & $-21,2$ & 17,1 & 1,2 & 3,6 & $-30,8$ \\
\hline $\mathrm{Fev}$ & 14,3 & 5,1 & 14,3 & 0,0 & 3,5 & $-31,4$ & 14,6 & 2,1 & 3,2 & $-37,3$ \\
\hline Mar & 15,9 & 5,3 & 16,3 & 2,5 & 2,9 & $-45,3$ & 16,4 & 3,1 & 2,9 & $-45,3$ \\
\hline Abr & 11,8 & 2,7 & 12,3 & 4,2 & 3,3 & 22,2 & 12,3 & 4,2 & 3,6 & 33,3 \\
\hline Mai & 9,4 & 4,1 & 10,2 & 8,5 & 3,4 & $-17,1$ & 9,4 & 0,0 & 3,6 & $-12,2$ \\
\hline Jun & 6,8 & 3,5 & 6,8 & 0,0 & 3,5 & 0,0 & 7,3 & 7,4 & 3,1 & $-11,4$ \\
\hline Jul & 7,2 & 3,4 & 7,1 & $-1,4$ & 2,7 & $-20,6$ & 7,0 & $-2,8$ & 2,9 & $-14,7$ \\
\hline Ago & 8,5 & 2,5 & 8,4 & $-1,2$ & 3,2 & 28,0 & 8,4 & $-1,2$ & 3,0 & 20,0 \\
\hline Set & 11,9 & 5,2 & 11,7 & $-1,7$ & 3,2 & $-38,5$ & 11,8 & $-0,8$ & 3,2 & $-38,5$ \\
\hline Out & 12,1 & 4,3 & 12,5 & 3,3 & 3,7 & $-14,0$ & 12,2 & 0,8 & 3,8 & $-11,6$ \\
\hline Nov & 13,5 & 4,1 & 13,3 & $-1,5$ & 3,4 & $-17,1$ & 13,7 & 1,5 & 3,1 & $-24,4$ \\
\hline Dez & 16,0 & 5,1 & 16,4 & 2,5 & 3,5 & $-31,4$ & 16,0 & 0,0 & 3,5 & $-31,4$ \\
\hline VMVP & & & & 2,2 & & 23,9 & & 2,1 & & 25,9 \\
\hline
\end{tabular}


média da probabilidade de um dia ser chuvoso tendo o anterior também sido chuvoso, assim como em relação ao desvio padrão.

$\mathrm{Na}$ Tabela 3 encontram-se os resultados relativos aos valores médios mensais da probabilidade de um dia ser chuvoso, tendo sido o anterior chuvoso e dos respectivos desvios padrão dos dados gerados pelo CLIMABR e pelo CLIGEN, bem como as variações percentuais e o valor médio destas variações, em relação aos dados observados na estação Capela Mayrink.

Com relação aos resultados estimados pelo modelo, as médias mensais apresentaram variações de - 14,0 (julho) a $0,0 \%$ (abril), resultado que ocorreu em pelo menos um mês em todas as estações, com exceção da estação Ilha dos Pombos. Os desvios padrão apresentaram variações percentuais que oscilaram entre uma superestimativa de 77,8 (outubro) a uma subestimativa de $36,0 \%$ (fevereiro).

As variações percentuais entre os resultados obtidos utilizando o CLIGEN e os valores observados apresentaram uma menor amplitude, variando de $-11,3$ (maio e agosto) a $1,6 \%$ (janeiro). Os desvios padrão obtidos pelo CLIGEN apresentaram maiores variações percentuais em relação aos dados observados do que os obtidos pelo CLIMABR, sendo que estes oscilaram de $-24,0$ até $80,0 \%$, o que ocorreu nos meses de fevereiro e maio, respectivamente. Os VMVP's apresentaram valores também bastante aproximados, sendo que o CLIMABR os apresentou ligeiramente superiores.

$\mathrm{Na}$ comparação dos resultados obtidos para as estações localizadas em regiões próximas ao litoral com os gerados nas estações localizadas em maiores latitudes, foram observados, mais uma vez, comportamentos diferenciados nos resultados, sobretudo em relação às médias geradas pelos modelos. Os maiores valores de VMVP para as médias mensais geradas pelo CLIMABR ocorreram nas estações localizadas em maiores altitudes, tendência que se repetiu também nos VMVP's das médias mensais geradas pelo CLIGEN. Com relação aos desvios padrão gerados pelo CLIMABR e pelo CLIGEN, os maiores valores de VMVP obtidos aconteceram, nos dois casos, na estação Tanguá. A estação Ilha Pombos foi a que apresentou o maior valor de VMVP para as médias, tanto no CLIMABR quanto no CLIGEN.

Probabilidade de um dia ser chuvoso tendo sido o anterior seco - P(W/D): Notou-se que, em geral, o CLIMABR e o CLIGEN apresentaram maiores variações percentuais em relação aos valores observados nos desvios padrão que nas médias mensais, semelhante ao que se notou nos resultados obtidos para $\mathrm{NW}$ e $\mathrm{P}(\mathrm{W} / \mathrm{W})$; outro comportamento evidenciado foi a tendência de obtenção de valores, tanto pelo CLIMABR como pelo CLIGEN, superiores aos observados com relação às médias mensais.

Os resultados relativos aos valores médios mensais da probabilidade de um dia ser chuvoso tendo sido o anterior seco e dos respectivos desvios padrão dos dados gerados pelo CLIMABR e pelo CLIGEN, e as variações percentuais em relação aos dados observados e o valor médio destas variações, para a estação ELETROBRÁS, encontra-se no Tabela 4.

No que diz respeito aos resultados gerados pelo CLIMABR, as médias mensais apresentaram variações de 0 (janeiro, junho e setembro) a 11,5\% (abril) e os desvios padrão apresentaram variações percentuais que oscilaram entre uma subestimativa de 33,3\% (setembro) a uma superestimativa de 33,3\% (junho).

As variações percentuais entre os resultados obtidos utilizando-se o CLIGEN e os valores observados apresentaram em comportamento semelhante ao CLIMABR, variando de 3,7 (janeiro) a 12,0\% (março); já os desvios padrão apresentaram variações percentuais em relação aos dados observados que oscilaram de $-28,6$ até $33,3 \%$, o que ocorreu nos meses de março e junho, respectivamente.

Levando-se em conta os VMVP's obtidos para o CLIMABR, vê-se que o maior valor da média mensal ocorreu na estação Posto Garrafão, sendo igual a 8,3\%. Na estação Tanguá foi gerado o maior VMVP para o desvio padrão igual a $31,5 \%$, enquanto para os dados gerados pelo CLIGEN os maiores valores de VMVP foram verificados nas estações Tocos e Tanguá, em que na primeira o VMVP foi de 7,5\% para a média

Tabela 3. Valores médios mensais da probabilidade de um dia ser chuvoso, tendo sido o anterior chuvoso $\overline{\mathrm{P}(\mathrm{W} / \mathrm{W})}$ e os respectivos desvios padrão (s) dos dados observados e gerados pelo CLIMABR e pelo CLIGEN, variações percentuais $(\delta)$ em relação aos dados observados e o valor médio das variações percentuais (VMVP) para a estação Capela Mayrink

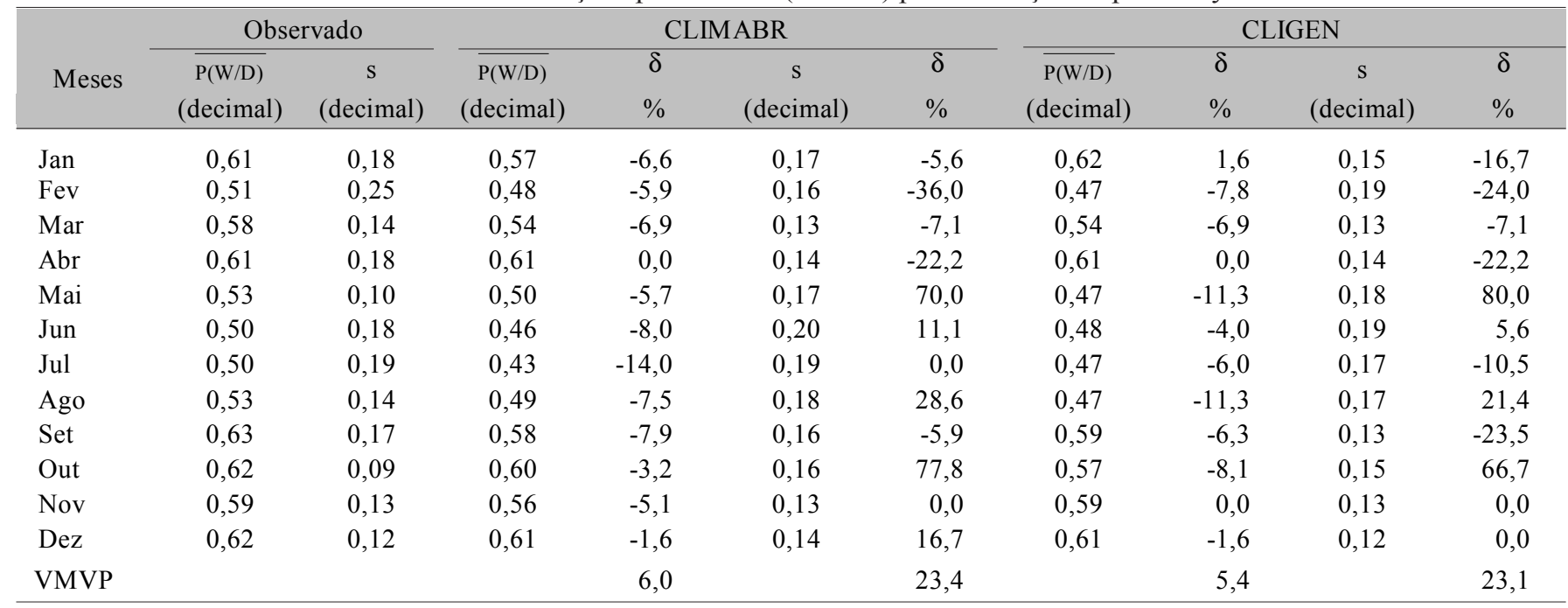


Tabela 4. Valores médios mensais da probabilidade de um dia ser chuvoso tendo sido o anterior seco [ $\overline{\mathrm{P}(\mathrm{W} / \mathrm{D})}]$ e os respectivos desvios padrão (s) dos dados observados e gerados pelo CLIMABR e pelo CLIGEN, variações percentuais $(\delta)$ em relação aos dados observados e o valor médio das variações percentuais (VMVP) para a estação ELETROBRÁS

\begin{tabular}{|c|c|c|c|c|c|c|c|c|c|c|}
\hline \multirow[b]{2}{*}{ Meses } & \multicolumn{2}{|c|}{ Observado } & \multicolumn{4}{|c|}{ CLIMABR } & \multicolumn{4}{|c|}{ CLIGEN } \\
\hline & $\begin{array}{c}\overline{\mathrm{P}(\mathrm{W} / \mathrm{D})} \\
\text { (decimal) }\end{array}$ & $\begin{array}{c}\mathrm{s} \\
(\text { decimal })\end{array}$ & $\begin{array}{c}\overline{\mathrm{P}(\mathrm{W} / \mathrm{D})} \\
\text { (decimal) }\end{array}$ & $\begin{array}{c}\delta \\
\%\end{array}$ & $\begin{array}{c}\mathrm{s} \\
\text { (decimal) }\end{array}$ & $\begin{array}{c}\delta \\
\%\end{array}$ & $\begin{array}{c}\overline{\mathrm{P}(\mathrm{W} / \mathrm{D})} \\
(\text { decimal) }\end{array}$ & $\begin{array}{l}\delta \\
\%\end{array}$ & $\begin{array}{c}\mathrm{s} \\
\text { (decimal) }\end{array}$ & $\begin{array}{l}\delta \\
\%\end{array}$ \\
\hline Fev & 0,22 & 0,12 & 0,23 & 4,5 & 0,10 & $-16,7$ & 0,24 & 9,1 & 0,10 & $-16,7$ \\
\hline Mar & 0,25 & 0,14 & 0,27 & 8,0 & 0,10 & $-28,6$ & 0,28 & 12,0 & 0,10 & $-28,6$ \\
\hline $\mathrm{Abr}$ & 0,26 & 0,10 & 0,29 & 11,5 & 0,11 & 10,0 & 0,28 & 7,7 & 0,12 & 20,0 \\
\hline Jun & 0,14 & 0,06 & 0,14 & 0,0 & 0,08 & 33,3 & 0,15 & 7,1 & 0,08 & 33,3 \\
\hline Jul & 0,15 & 0,07 & 0,16 & 6,7 & 0,08 & 14,3 & 0,16 & 6,7 & 0,07 & 0,0 \\
\hline Ago & 0,20 & 0,07 & 0,21 & 5,0 & 0,09 & 28,6 & 0,22 & 10,0 & 0,08 & 14,3 \\
\hline Set & 0,26 & 0,15 & 0,26 & 0,0 & 0,10 & $-33,3$ & 0,27 & 3,8 & 0,11 & $-26,7$ \\
\hline Out & 0,34 & 0,12 & 0,37 & 8,8 & 0,14 & 16,7 & 0,36 & 5,9 & 0,12 & 0,0 \\
\hline Nov & 0,31 & 0,11 & 0,34 & 9,7 & 0,13 & 18,2 & 0,34 & 9,7 & 0,13 & 18,2 \\
\hline
\end{tabular}

mensal, enquanto que na estação Tanguá o desvio padrão apresentou VMVP de 27,0\%.

Considerando-se os resultados obtidos para $\mathrm{P}(\mathrm{W} / \mathrm{D})$, não se evidenciou tendência de comportamento em relação à altitude das estações, mas os maiores valores de VMVP para as médias mensais geradas pelo CLIMABR e pelo CLIGEN, ocorreram nas estações localizadas em maiores altitudes, tendência que não se repetiu para os VMVP's dos desvios padrão gerados pelos dois modelos. Quanto aos desvios padrão gerados pelo CLIMABR e pelo CLIGEN, os maiores valores obtidos ocorreram, nos dois casos, na estação Tanguá, seguida pelas estações Posto Garrafão e Ilha dos Pombos; no entanto, não se constatou predominância dos maiores valores para as estações localizadas na região serrana.

\section{Precipitação total diária $-\mathbf{P}$}

Os valores observados da precipitação total diária para a estação Santa Cecília, bem como os dados gerados pelo CLIMABR e pelo CLIGEN, são apresentados na Figura 2.
A tendência de superestimativa das médias mensais e subestimativas dos desvios padrão, com relação aos dados observados, é observada nos resultados obtidos pelos dois modelos.

A partir da comparação dos resultados gerados pelo CLIMABR com os dados observados, é possível constatar que as maiores variações percentuais das médias mensais da precipitação total diária ocorreram em abril e julho, onde os valores observados foram superestimados em 3,3\%.

Com relação aos desvios padrão obtidos pelo CLIMABR, nos meses de maio e julho foram observadas, respectivamente, subestimativas iguais a 2,8 e 3,1\%. O CLIMABR gerou valores de VMVP menores que os estimados pelo CLIGEN; o valor de VMVP para as médias mensais foi igual a 2,2\% e, para o desvio padrão, de 1,9\%. Nesta mesma estação o CLIGEN apresentou um desempenho inferior ao modelo CLIMABR com relação às médias mensais calculadas e aos desvios padrão estimados. Em dez meses, os resultados gerados pelo CLIMABR para a média mensal da precipitação total diária foram melhores quando

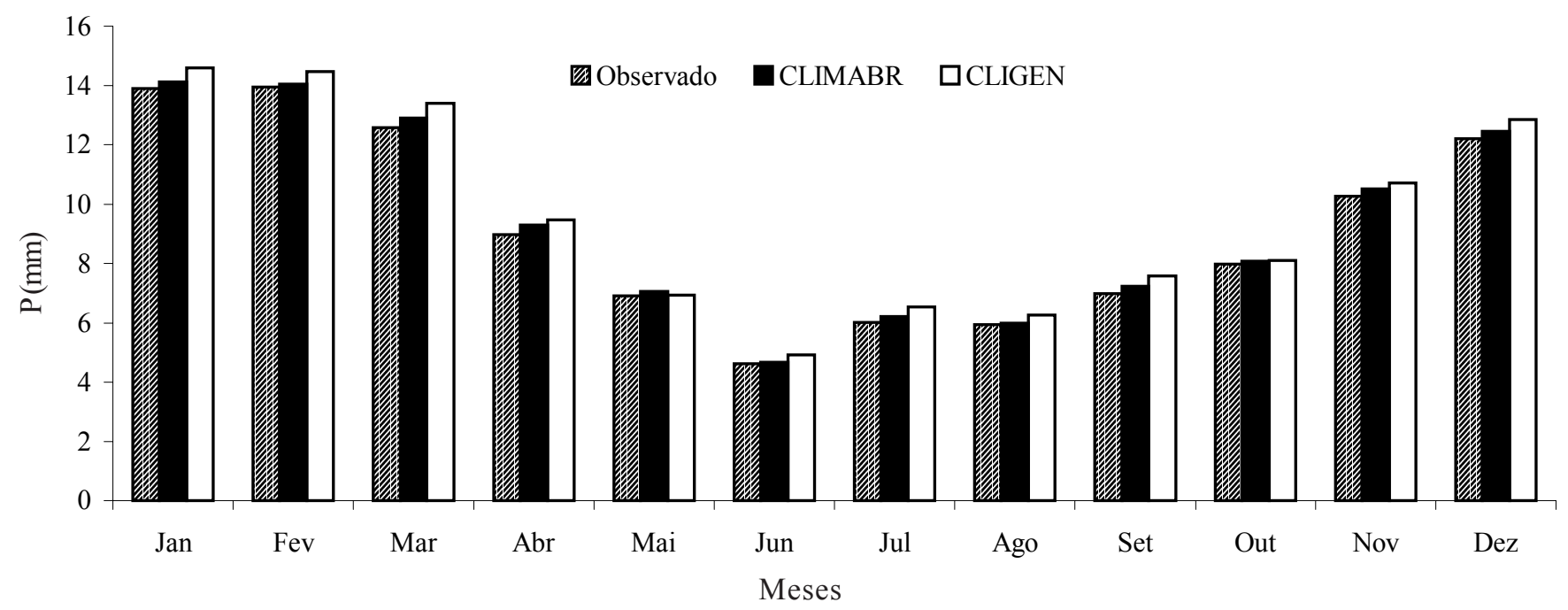

Figura 2. Distribuição mensal da precipitação total diária ( P ) para a estação Santa Cecília. 
comparados com os estimados pelo CLIGEN; e em 11 meses, o CLIMABR superou o CLIGEN quando considerados os desvios padrão gerados.

A Figura 3 mostra a correlação entre os dados observados e gerados pelo modelo para a mesma estação. A análise da equação de regressão entre o $\mathrm{P}_{\text {CLIMABR }}$ e o $\mathrm{P}_{\text {observado }}$ confirma a tendência de superestimativa observada anteriormente (coeficiente angular de 1,0194).

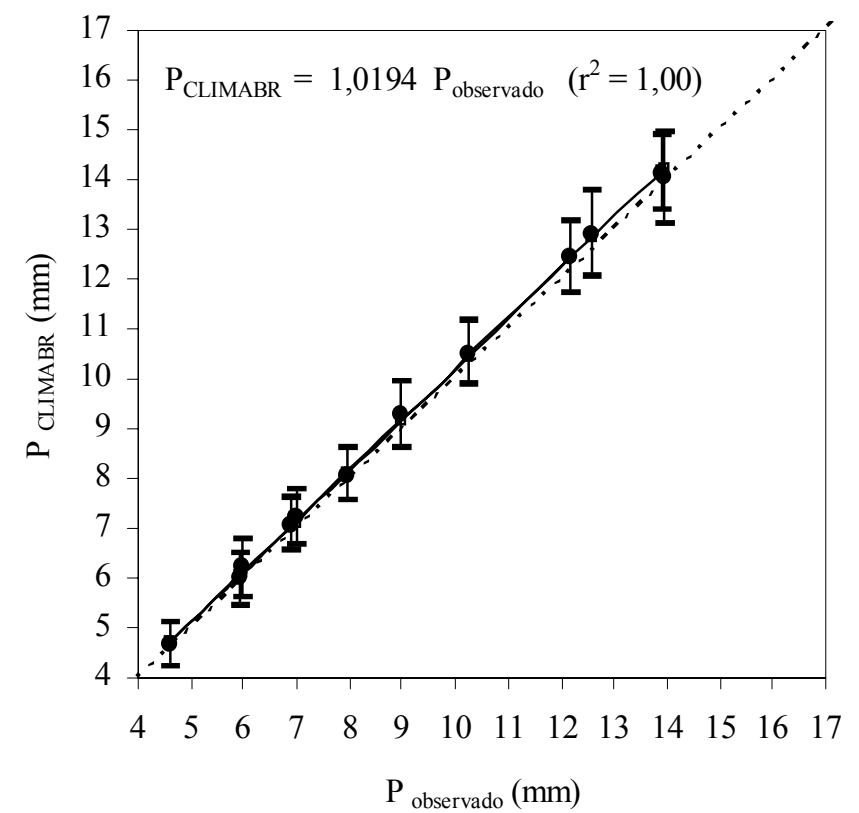

Figura 3. Comparação da média mensal da precipitação total diária (P) observada com a gerada pelo modelo na estação Santa Cecília, associado a um intervalo de confiança de 95\%.

Obteve-se um coeficiente de determinação igual a 1,00, o que indica também uma correlação adequada entre os dados observados e os gerados pelo modelo; este resultado é confirmado pelas barras de erros, onde se interceptam, simultaneamente, as linhas de equivalência e de regressão.

Comparando-se os resultados obtidos pelo CLIMABR, com os do CLIGEN, pode-se constatar que os melhores resultados foram obtidos com o uso do CLIMABR, tanto para as médias mensais como para os desvios padrão.

Um fato importante a relatar é que o CLIGEN apresentou inconsistências nas médias mensais simuladas para os meses de outubro, novembro e dezembro, para a estação ELETROBRÁS, em que o modelo americano gerou valores iguais a $0,3 \mathrm{~mm}$ para média mensal de precipitação nos três meses, enquanto os dados observados registraram valores de $7,8,9,4$ e $14,0 \mathrm{~mm}$.

\section{CONCLUSÕES}

A comparação dos dados gerados pelo CLIMABR com as observações do número de dias chuvosos, das probabilidades $\mathrm{P}(\mathrm{W} / \mathrm{W})$ e $\mathrm{P}(\mathrm{W} / \mathrm{D})$, além da precipitação total diária, mostrou bom desempenho para a estimativa dessas variáveis. Os resultados obtidos permitiram concluir-se que:
1. Para o número de dias chuvosos tanto o CLIMABR quanto o CLIGEN apresentaram estimativas próximas aos valores observados.

2. O CLIMABR subestimou a média mensal da probabilidade de um dia ser chuvoso tendo sido o dia anterior chuvoso $\mathrm{P}(\mathrm{W} /$ W). entretanto, a tendência não foi observada para o desvio padrão desta variável.

3. Para a probabilidade de um dia ser chuvoso tendo sido o anterior seco $\mathrm{P}(\mathrm{W} / \mathrm{D})$ foi evidenciada uma tendência do CLIMABR em superestimar as médias mensais.

4. Foi evidenciada uma tendência do CLIMABR em superestimar as variações das médias mensais e subestimar as variações do desvio padrão observadas para a precipitação total diária.

\section{AGRADECIMENTOS}

Ao Centro Federal de Educação Tecnológica de Campos CEFET Campos e ao Parque de Alta Tecnologia Norte Fluminense - TECNORTE, pela oportunidade concedida para a realização deste trabalho. À Fundação Superintendência Estadual de Rio e Lagoas - SERLA e à Companhia LIGHT Serviços de Eletricidade S.A, pela cessão dos dados que permitiram a execução desta pesquisa.

\section{LITERATURA CITADA}

Elliot, W.J.; Arnold, C.D. Validation of the weather generator CLIGEN with precipitation data from Uganda. Transactions of the ASAE, St. Joseph, v.44, n.1, p.53-58, 2001.

Flanagan, D.C.; Meyer, C.R.; Yu, B., Scheele, D.I. Evaluation and enhacement of the CLIGEN weather generator. In: International Symposium of ASAE and Soil Erosion Research for the 21 Century, 2001, Honolulu, 2001 . p.107110.

Nicks, A.D.; Lane, L.J.; Gander, G.A. Weather generator: USDAWater erosion prediction project (WEPP). West Lafayette: USDA-ARS National Soil Erosion Research Laboratory, 1995. cap.2, p. 22 .

Virgens Filho, J.S. Ferramenta computacional para simulação de séries climáticas diárias, baseada na parametrização dinâmica das distribuições de probabilidade. Botucatu: UNESP, 2001.92p. Tese Doutorado

Virgens Filho, J.S. Modelo computacional para simulação de dados climáticos. Botucatu: UNESP, 1997. 86p. Dissertação Mestrado

Wilks, D.S. Interannual variability and extreme-value characteristics of several stochastic daily precipitation models. Agricultural and Forest Meteorology, Ithaca, v. 93, p.153-169, 1999.

$\mathrm{Yu}$, B. Improvement and evaluation of CLIGEN for storm generation. Transactions of the ASAE, St. Joseph, v.43, n.2, p.301-307, 2001. 